\title{
Association between Atherogenic Index of Plasma and the Risk of Obstructive Sleep Apnea in Korean Adults Who Admitted to the Sleep Clinic
}

\author{
Sei Won Kim', Sang Haak Lee', and Hyeon Hui Kang² \\ 'Division of Pulmonary, Critical Care and Sleep Medicine, Eunpyeong St. Mary's Hospital, College of Medicine, \\ The Catholic University of Korea, Seoul, Korea \\ ${ }^{2}$ Division of Pulmonary, Critical Care and Sleep Medicine, Ulsan University Hospital, University of Ulsan College of Medicine, Ulsan, Korea
}

\begin{abstract}
Objective: Atherogenic index of plasma (AIP) is closely related to insulin resistance, which is a major characteristics of obstructive sleep apnea (OSA). However, data on the association between AIP and OSA has been limited. This study evaluated the association of AIP with OSA. Methods: AIP was assessed in 180 consecutive Korean adults with suspected OSA who admitted to the sleep clinic between 2010 and 2012. OSA was defined as the occurrence of more than 5 apnea-hypopnea index (AHI) events/h. All participants were divided into three groups according to the AIP tertiles, which were determined by the log of (triglyceride/high-density lipoprotein cholesterol). Results: The prevalence of OSA increased [group I (lowest): 73.8\%; group II: 86.4\%; group III (highest): $91.7 \%$ ] as increasing AIP tertiles ( $p=0.022$ ). AIP had a positive correlation with $\mathrm{AHI}(r=0.210 ; p=0.005)$. In univariate linear regression analysis, AIP (per 0.1 -unit increase) was significantly related to $\mathrm{AHI}$ $(\beta=2.460 ; p=0.005)$. Univariate logistic regression analysis showed that AIP was associated with an increased risk of OSA [odds ratio (OR): 1.450; $95 \%$ confidence interval (CI): 1.179-1.785; $p<0.001$ ]. However, this association was attenuated after adjusting for multiple risk factors including age, male sex, alcohol consumption, obesity, and smoking (OR: 1.252; $95 \% \mathrm{Cl}$ : 0.995-1.575; p=0.055). Conclusion: Despite a significant correlation of AIP with AHI, AIP was not an independent predictive marker for OSA beyond multiple risk factors. Further investigation with larger sample size might be necessary.
\end{abstract}

Key Words: Atherogenic index of plasma; Obstructive sleep apnea; Apnea-hypopnea index

Received: November 24, 2020 Revised: December 7, 2020 Accepted: December 8, 2020

Corresponding author: Hyeon Hui Kang, MD, PhD, Division of Pulmonary, Critical Care and Sleep Medicine, Ulsan University Hospital, University of Ulsan College of Medicine, 877 Bangeojinsunhwando-ro, Dong-gu, Ulsan 44033, Korea.

Tel: 82-52-250-8660, Fax: 82-52-250-7048, E-mail: khh3822@naver.com

(a) This is an Open Access article distributed under the terms of the Creative Commons Attribution Non-Commercial License (https://creativecommons.org/licenses/bync/4.0) which permits unrestricted non-commercial use, distribution, and reproduction in any medium, provided the original work is properly cited.

\section{INTRODUCTION}

Obstructive sleep apnea (OSA) is a major health problem which is closely related to arousal, sympathetic activation, and oxygen desaturation during sleep. The prevalence of OSA in the general population is estimated to be $9 \%$ to $38 \%$; OSA is more common in males, the elderly, and obese individuals relative to the general population [1]. It is well-established that OSA contributes to the increase of cardiovascular morbidity and mortality $[2,3]$.

Subjects with OSA display clinical features similar to those with metabolic syndrome which represents insulin resistance as major characteristics [4,5]. Recently, the atherogenic index of plasma
(AIP), which is calculated by log [triglyceride/high-density lipoprotein cholesterol (HDL-C)] [6], has been considered as a useful marker for plasma atherogenicity based on its significant association with lipoprotein particle size, cholesterol esterification rates, and remnant lipoproteinemia [6-8]. In addition, the AIP is closely correlated with the degree of insulin resistance [9]. However, data regarding the significance of AIP on OSA had been limited in clinical practice. In the present study, we aimed to evaluate the association between AIP and OSA in Korean adults with suspected OSA. 


\section{METHODS}

\section{Subjects and study design}

This study retrospectively analyzed clinical characteristics of 180 consecutive patients referred to the sleep clinic for polysomnography examinations at St. Paul's Hospital between January 2010 and October 2012. All patients had at least one symptom suggesting OSA, including snoring, excessive daytime sleepiness, witnessed apneic incidents, and nocturnal choking. Overnight polysomnographic examination with a SomnoStar Pro 7-3a system (Cardinal Health, Inc., Dublin, OH, USA) was performed on all participants. Electroencephalographic, electrooculographic, electrocardiographic, and electromyographic surface electrodes were used to record participant data. Nasal and oral airflow, tracheal sounds, thoracic and abdominal movement, and positional changes during sleep were also recorded. Transcutaneous peripheral oxygen saturation $\left(\mathrm{SpO}_{2}\right)$ was monitored with a pulse oximeter. After data collection using a computerized polysomnographic system, a manual scoring was performed. Rechtschaffen and Kales criteria [10] was used to define sleep status. The criteria of the American Academy of Sleep Medicine were applied for respiratory events [11]. Airflow reduction of $\geq 90 \%$ of baseline values for $\geq 10$ s was defined as apnea. Events involving $\geq 30 \%$ airflow reduction for $\geq 10 \mathrm{~s}$ accompanied by $\mathrm{a} \geq 3 \%$ drop in oxygen saturation or arousal were defined as hypopnea. Apnea-hypopnea index (AHI) was defined as the number of apnea or hypopnea events per hour that occurred during sleep. OSA was defined as the occurrence of $\geq 5 \mathrm{AHI}$ events/h; the severity was defined as mild (5 $\leq \mathrm{AHI}<15)$, moderate $(15 \leq \mathrm{AHI}<30)$, and severe $(\mathrm{AHI} \geq 30)$ based on the number of events per night of sleep [12]. All blood samples were obtained after at least $8 \mathrm{~h}$ fasting. All subjects completed a questionnaire about alcohol consumption and smoking history. Alcohol intake was calculated based on the daily recall of consumption. Regardless of the type of alcohol, alcohol consumption of less than 12 cups in one's lifetime was considered to have no alcohol history. Smokers reported a smoking history of at least 2 years, currently smoked more than 20 cigarettes/day. Ex-smokers reported a history of past exposure to nicotine ( $\geq 5$ cigarettes in lifetime), but no current smoking. Never-smokers smoked fewer than five cigarettes in their lifetimes. Body mass index (BMI) was defined as the patient's weight $(\mathrm{kg}) / \mathrm{height}\left(\mathrm{m}^{2}\right)$. Obesity was defined as a BMI of $\geq 25 \mathrm{~kg} / \mathrm{m}^{2}$ based on the cut-off values for Asians. Excessive daytime sleepiness was defined as Epworth sleepiness scale score $>10$ [13]. The Institutional Review Board of St. Paul's Hospital approved the protocol (PC14OISI0059), and all participants provided written informed consent.

\section{Statistical analysis}

Continuous and categorical variables are presented as mean \pm standard deviation and number (percentage), respectively. Oneway analysis of variance was used to compare continuous variables. The $\chi^{2}$-test or Fisher's exact test was used to compare categorical variables. Pearson's correlation test was used to evaluate the correlation between variables. Univariate linear regression analysis was used to identify the association between clinical variables and AHI. Univariate and multivariate logistic regression analyses were performed to identify independent risk factors for OSA. The forced entry method was used to enter independent variables into the multiple regression analysis. All statistical analyses were performed with the Statistical Package for the Social Sciences version 19 (IBM Corp., Armonk, NY, USA). p values $<0.05$ were considered statistically significant.

\section{RESULTS}

Baseline characteristics of all participants are included in Table 1. The mean age of the participants (133 males, $73.9 \%$ ) was $48.6 \pm 13.8$ years. Mean BMI values were $26.4 \pm 4.1 \mathrm{~kg} / \mathrm{m}^{2}$; and the prevalence of obesity was $59.4 \%$. The prevalence of alcohol consumption and smoking were $61.1 \%$ and $37.2 \%$, respectively. The mean levels of total cholesterol, triglyceride, HDL-C, and low-density lipoprotein cholesterol (LDL-C) $(\mathrm{mg} / \mathrm{dL})$ were $186.3 \pm 39.7,163.2 \pm 94.0$, 47.6 \pm 11.5 , and 113.6 \pm 35.6 , respectively. Mean AIP values were $0.50 \pm 0.24$. The mean AHI levels determined for all study participants were $31.6 \pm 28.3 / \mathrm{h}$, and the overall prevalence of OSA was $83.9 \%$.

The prevalence of OSA significantly increased as AIP tertile increased [group I (lowest): 73.8\%; group II: $86.4 \%$; group III (highest): $91.7 \% ; p=0.022$ ] (Figure 1). The presence and severity of OSA associated with AIP tertiles are shown in Figure 2. AIP had a sig-

Table 1. Baseline characteristics of study subjects

\begin{tabular}{|c|c|}
\hline Characteristics & $\mathrm{n}=180$ \\
\hline Age (yr) & $48.6 \pm 13.8$ \\
\hline Male & $133(73.9)$ \\
\hline BMI & $26.4 \pm 4.1$ \\
\hline Obesity & $107(59.4)$ \\
\hline Alcohol consumption & $110(61.1)$ \\
\hline Smoking & $67(37.2)$ \\
\hline Total cholesterol $(\mathrm{mg} / \mathrm{dL})$ & $186.3 \pm 39.7$ \\
\hline Triglyceride (mg/dL) & $163.2 \pm 94.0$ \\
\hline $\mathrm{HDL}-\mathrm{C}(\mathrm{mg} / \mathrm{dL})$ & $47.6 \pm 11.5$ \\
\hline $\mathrm{LDL}-\mathrm{C}(\mathrm{mg} / \mathrm{dL})$ & $113.6 \pm 35.6$ \\
\hline OSA & $151(83.9)$ \\
\hline Epworth sleepiness scale & $9.4 \pm 4.3$ \\
\hline Lowest $\mathrm{SpO}_{2}(\%)$ & $80.8 \pm 8.7$ \\
\hline $\mathrm{AHI}(/ \mathrm{h})$ & $31.6 \pm 28.3$ \\
\hline Sleep period time $(\mathrm{min})$ & $414.8 \pm 52.1$ \\
\hline Total sleep time (min) & $347.3 \pm 67.8$ \\
\hline Sleep efficiency (\%) & $81.3 \pm 14.5$ \\
\hline Sleep latency (min) & $13.1 \pm 28.5$ \\
\hline AIP & $0.50 \pm 0.24$ \\
\hline
\end{tabular}

Values are given as the mean \pm standard deviation or number (\%). AHI apnea-hypopnea index, AIP: atherogenic index of plasma, BMI: body mass index, HDL-C: high-density lipoprotein cholesterol, LDL-C: lowdensity lipoprotein cholesterol, OSA: obstructive sleep apnea, $\mathrm{SpO}_{2}$ : peripheral oxygen saturation 
nificant correlation with $\mathrm{AHI}$ in all participants $(\mathrm{r}=0.210 ; \mathrm{p}=0.005)$ (Figure 3). Univariate linear regression analysis showed that alcohol consumption $(\beta=11.796 ; p=0.006)$, obesity $(\beta=23.641 ; p<0.001)$, smoking $(\beta=14.326 ; p=0.001)$, and AIP (per 0.1-unit increase) $(\beta=$ $2.460 ; \mathrm{p}=0.005$ ) had a significant association with AHI (Table 2).

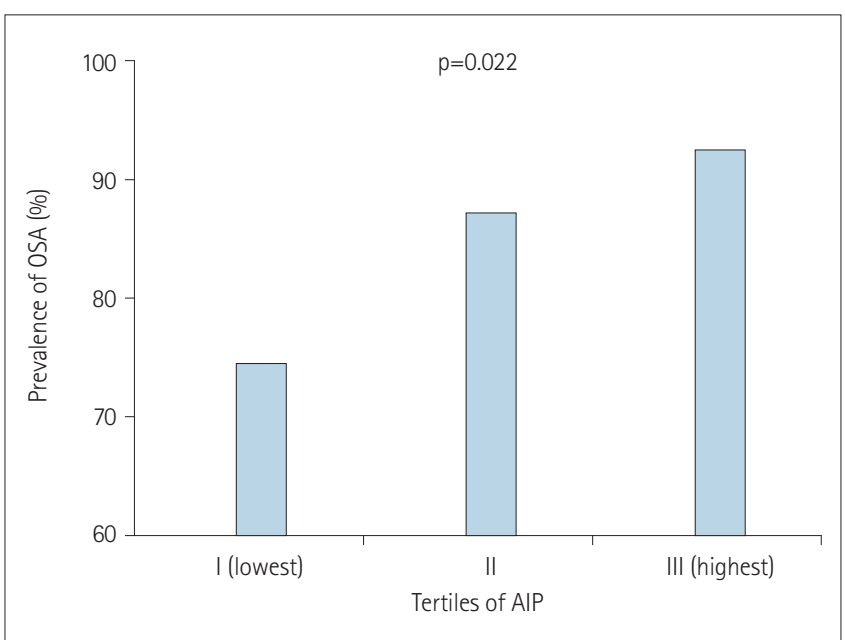

Figure 1. Comparison of obstructive sleep apnea (OSA) prevalence according to atherogenic index of plasma (AIP) tertiles.

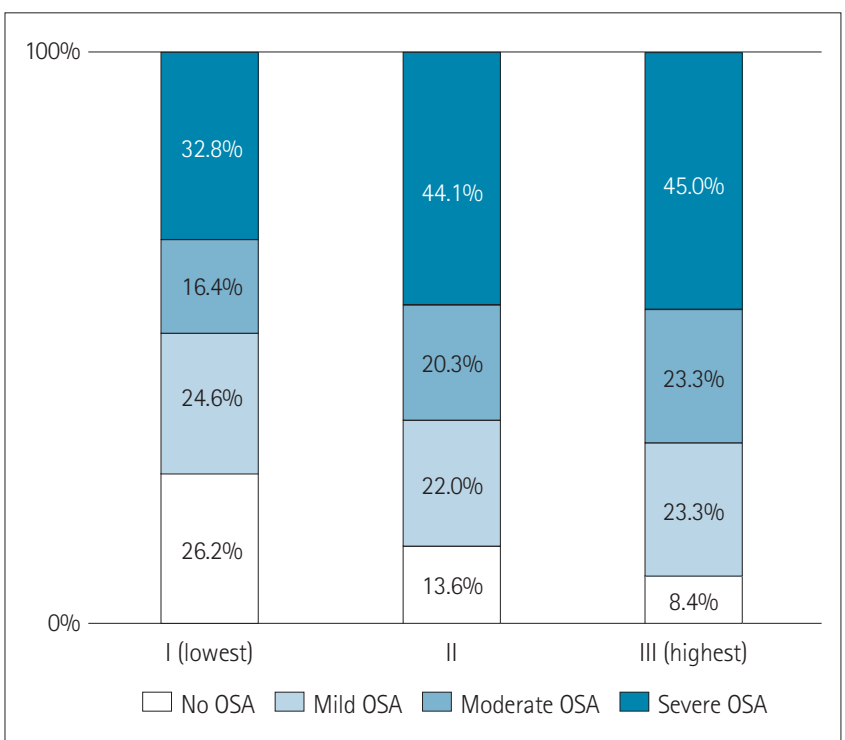

Figure 2. Composition of the presence and severity of obstructive sleep apnea (OSA) according to atherogenic index of plasma (AIP) tertiles.
Univariate logistic regression analysis showed that age (per 1-year increase) [odds ratio (OR): 1.040; 95\% confidence interval (CI): 1.009-1.072; $\mathrm{p}=0.011$ ], male sex (OR: 2.796; 95\% CI: $1.225-$ 6.383; $\mathrm{p}=0.015$ ), obesity (OR: 4.998; 95\% CI: 2.071-12.058; $\mathrm{p}<$ 0.001), smoking (OR: 3.344; 95\% CI: 1.210-9.242; $\mathrm{p}=0.020$ ), and AIP (per 0.1 unit increase) (OR: 1.450; 95\% CI: 1.179-1.785; p< $0.001)$ were associated with an increased risk of OSA. In multivariate logistic regression analysis, age (OR: 1.054; 95\% CI: $1.018-$ 1.093; $\mathrm{p}=0.003$ ), male sex (OR: 3.132; 95\% CI: 1.063-9.224; $\mathrm{p}=$ 0.038), and obesity (OR: 3.621; 95\% CI: 1.366-9.599; $\mathrm{p}=0.010$ ) were independently associated with an increased risk of OSA; however, AIP did not have independent association with the risk OSA (OR: 1.252; 95\% CI: 0.995-1.575; p=0.055) (Table 3).



Figure 3. Correlation between atherogenic index of plasma (AIP) and apnea-hypopnea index $(\mathrm{AHI})$.

Table 2. Association between clinical variables and $\mathrm{AHI}$

\begin{tabular}{lccr} 
& $\beta$ & $\mathrm{SE}$ & $\mathrm{p}$ \\
Age, per 1-years & 0.105 & 0.153 & 0.493 \\
Male & 9.163 & 4.754 & 0.056 \\
Alcohol consumption & 11.796 & 4.237 & 0.006 \\
Obesity & 23.641 & 3.915 & $<0.001$ \\
Smoking & 14.326 & 4.231 & 0.001 \\
AIP, per 0.1-unit & 2.460 & 0.857 & 0.005 \\
\hline
\end{tabular}

AHI: apnea-hypopnea index, AIP: atherogenic index of plasma

Table 3. Multivariate logistic regression analysis for the impact of clinical variables on OSA

\begin{tabular}{|c|c|c|c|c|}
\hline \multirow{2}{*}{ Variables } & \multicolumn{2}{|c|}{ Univariate } & \multicolumn{2}{|c|}{ Multivariate } \\
\hline & OR (95\% CI) & $\mathrm{p}$ & OR $(95 \% \mathrm{CI})$ & $\mathrm{p}$ \\
\hline Age, per 1-year & $1.040(1.009-1.072)$ & 0.011 & $1.054(1.018-1.093)$ & 0.003 \\
\hline Male & $2.796(1.225-6.383)$ & 0.015 & $3.132(1.063-9.224)$ & 0.038 \\
\hline Alcohol consumption & $1.870(0.840-4.163)$ & 0.125 & $0.892(0.317-2.507)$ & 0.828 \\
\hline Obesity & $4.998(2.071-12.058)$ & $<0.001$ & $3.621(1.366-9.599)$ & 0.010 \\
\hline Smoking & $3.344(1.210-9.242)$ & 0.020 & $2.065(0.631-6.758)$ & 0.230 \\
\hline AIP, per 0.1-unit & $1.450(1.179-1.785)$ & $<0.001$ & $1.252(0.995-1.575)$ & 0.055 \\
\hline
\end{tabular}

AIP: atherogenic index of plasma, CI: confidence interval, OR: odds ratio, OSA: obstructive sleep apnea 


\section{DISCUSSION}

To the best of our knowledge, this is the first study to evaluate the association of AIP with the risk of OSA in Korean adults who suspected OSA. In the present study, AIP had significant relationship with AHI together with other clinical factors including alcohol consumption, obesity, and smoking. Although subjects with higher AIP levels were more prone to have the increased risk of OSA, AIP was not an independent risk factor for OSA beyond other risk factors.

AIP is inversely and significantly correlated with measures of insulin sensitivity. In addition, AIP has been reported to correlate with insulin resistance [14]. High levels of the triglycerides to HDL-C ratio have been associated with metabolic syndrome $[15,16]$. This might be related to the fact that hypertriglyceridemia stimulates the activity of cholesteryl ester transfer protein, which exchanges triglycerides from triglyceride-rich lipoproteins for cholesteryl esters from low- and high-density lipoproteins [17]. Triglycerides enrichment of low- and high-density lipoprotein particles renders them better substrates for lipolysis by hepatic lipase, resulting in high-density lipoprotein catabolism and elimination and the formation of more numerous, denser low-density lipoprotein particles. Thus, AIP is an important parameter which can reflect the abnormal metabolism of both lipids and glucose.

Recent study reported that the co-occurrence of hypertriglyceridemia and OSA was affected by genetics, and heritable factors might play a crucial role in dyslipidemia pathogenesis in OSA [18]. Drager et al. [19] reported that OSA was strongly associated with metabolic syndrome components, especially triglyceride and glucose levels. These studies indicated that triglyceride or triglyceride-based combined indices such as AIP and triglyceride glucose (TyG) index could be an independent predictive marker for OSA. In the present study, unlike AIP, a significant association between triglyceride and AHI was not observed $(\beta=0.034 ; \mathrm{p}=0.128)$. Although higher AIP levels were significantly associated with an increased risk of OSA, its significance was not observed after adjusting for other clinical risk factors. This might be related to the small sample size of this cohort registry. However, recent study which analyzed the same registry data found that TyG index was determined to be independently associated with the risk of OSA [20]. This finding means that the predictive value of triglyceride-based combined indices for OSA might be somewhat different in adults with suspected OSA.

The present study had some limitations. First, clinical factors not considered could influence the study results because of observational design. Second, the population had an unbalanced sex distribution; more males than females were referred to sleep clinics. Third, we could not control the possible effects of underlying medications on OSA. Finally, the present results should be applied with caution to the general population because only patients highly suspected of having OSA were included.

In this observational cohort study, AIP had a significant and positive correlation with AHI. Although higher AIP levels tend- ed to be associated with the increased risk of OSA, AIP did not have an independent predictive value for OSA after adjusting for multiple clinical factors. Further prospective and longitudinal studies with large sample size should be necessary to confirm the value of the AIP for predicting OSA.

Acknowledgments

None

\section{Conflicts of Interest}

The authors have no potential conflicts of interest to disclose.

\section{Author Contributions}

Conceptualization: Hyeon Hui Kang. Data curation: Sei Won Kim. Formal analysis: Hyeon Hui Kang. Investigation: Sei Won Kim. Methodology: Hyeon Hui Kang, Sang Haak Lee. Project administration: Hyeon Hui Kang. Resources: Sei Won Kim, Sang Haak Lee. Supervision: Hyeon Hui Kang. Visualization: Hyeon Hui Kang, Sang Haak Lee. Writing—original draft: Sei Won Kim. Writing_ review \& editing: Hyeon Hui Kang, Sang Haak Lee.

\section{ORCID iDs}

\section{Sei Won Kim (1)}

https://orcid.org/0000-0002-2798-421X

Sang Haak Lee (1)

https://orcid.org/0000-0001-6259-7656

Hyeon Hui Kang (1)

https://orcid.org/0000-0001-5860-9407

\section{REFERENCES}

1. Senaratna CV, Perret JL, Lodge CJ, Lowe AJ, Campbell BE, Matheson MC, et al. Prevalence of obstructive sleep apnea in the general population: a systematic review. Sleep Med Rev 2017;34:70-81.

2. Choi JW, Song JS, Lee YJ, Won TB, Jeong DU. Increased mortality in relation to insomnia and obstructive sleep apnea in Korean patients studied with nocturnal polysomnography. J Clin Sleep Med 2017;13:49-56.

3. Leão S, Conde B, Fontes P, Calvo T, Afonso A, Moreira I. Effect of obstructive sleep apnea in acute coronary syndrome. Am J Cardiol 2016;117:10841087.

4. Expert Panel on Detection, Evaluation, and Treatment of High Blood Cholesterol in Adults. Executive summary of the third report of the National Cholesterol Education Program (NCEP) expert panel on detection, evaluation, and treatment of high blood cholesterol in adults (Adult Treatment Panel III). JAMA 2001;285:2486-2497.

5. Grundy SM, Cleeman JI, Daniels SR, Donato KA, Eckel RH, Franklin BA, et al. Diagnosis and management of the metabolic syndrome: an American Heart Association/National Heart, Lung, and Blood Institute Scientific Statement. Circulation 2005;112:2735-2752.

6. Dobiásová M, Frohlich J. The plasma parameter log (TG/HDL-C) as an atherogenic index: correlation with lipoprotein particle size and esterification rate in apoB-lipoprotein-depleted plasma (FER(HDL)). Clin Biochem 2001; 34:583-588

7. Dobiásová M. AIP--atherogenic index of plasma as a significant predictor of cardiovascular risk: from research to practice. Vnitr Lek 2006;52:64-71.

8. Quispe R, Manalac RJ, Faridi KF, Blaha MJ, Toth PP, Kulkarni KR, et al. Relationship of the triglyceride to high-density lipoprotein cholesterol (TG/HDLC) ratio to the remainder of the lipid profile: the Very Large Database of Lipids-4 (VLDL-4) study. Atherosclerosis 2015;242:243-250.

9. Tan MH, Johns D, Glazer NB. Pioglitazone reduces atherogenic index of 
plasma in patients with type 2 diabetes. Clin Chem 2004;50:1184-1188.

10. Rechtschaffen A, Kales A. A manual of standardized terminology, techniques and scoring system for sleep stages of human subjects. Los Angeles: Brain Information Service, Brain Research Institute; 1968.

11. Berry RB, Brooks R, Gamaldo CE, Harding SM, Marcus CL, Vaughn BV. The AASM manual for the scoring of sleep and associated events: rules, terminology and technical specifications, version 2.0. Darien: American Academy of Sleep Medicine; 2012.

12. McNicholas WT. Diagnosis of obstructive sleep apnea in adults. Proc Am Thorac Soc 2008;5:154-160.

13. Johns MW. A new method for measuring daytime sleepiness: the Epworth sleepiness scale. Sleep 1991;14:540-545.

14. Tan MH, Jones D, Willis B, Smith G, Andreou P. Atherogenic index of plasma in Bermudians with diabetes. Diabetes Res Clin Pract 2000;50(suppl 1): S132.

15. González-Chávez A, Simental-Mendía LE, Elizondo-Argueta S. Elevated triglycerides/HDL-cholesterol ratio associated with insulin resistance. Cir Cir 2011;79:126-131.
16. Karelis AD, Pasternyk SM, Messier L, St-Pierre DH, Lavoie JM, Garrel D, et al. Relationship between insulin sensitivity and the triglyceride-HDL-C ratio in overweight and obese postmenopausal women: a MONET study. Appl Physiol Nutr Metab 2007;32:1089-1096.

17. Guérin M, Le Goff W, Lassel TS, Van Tol A, Steiner G, Chapman MJ. Atherogenic role of elevated CE transfer from HDL to VLDL(1) and dense LDL in type 2 diabetes: impact of the degree of triglyceridemia. Arterioscler Thromb Vasc Biol 2001;21:282-288.

18. Meszaros M, Tarnoki AD, Tarnoki DL, Kovacs DT, Forgo B, Lee J, et al. Obstructive sleep apnea and hypertriglyceridaemia share common genetic background: results of a twin study. J Sleep Res 2020;29:e12979.

19. Drager LF, Lopes HF, Maki-Nunes C, Trombetta IC, Toschi-Dias E, Alves MJ, et al. The impact of obstructive sleep apnea on metabolic and inflammatory markers in consecutive patients with metabolic syndrome. PLoS One 2010;5:e12065.

20. Kang HH, Kim SW, Lee SH. Association between triglyceride glucose index and obstructive sleep apnea risk in Korean adults: a cross-sectional cohort study. Lipids Health Dis 2020;19:182. 